\title{
Enhanced Event Coverage and Redundant Data Minimization Employing Pentagonal Scalar Premier Selection in Wireless Multimedia Sensor Networks (WMSNs)
}

\author{
Sushree Bibhuprada B. Priyadarshini
}

\begin{abstract}
In the modern era, data redundancy has become one among the predominant ultimatums encountered in Wireless Multimedia Sensor Networks (WMSNs), which occurs because of event information reporting through the scalars residing at the superimposing zones of field of views (FoVs) of multiple camera sensors. As a result, same data is transferred many times, thus leading to redundancy in data transfer. Therefore, the aim is to select the representatives of scalar sensors called scalar premiers (SPs) that can report the event information in lieu of all the scalars while diminishing the redundant data transfer and improving the event coverage. We have proffered a pentagonal scheme of SP selection that chooses five SPs in each of the virtual compartments of the monitored zone efficiently. The chosen SPs operate as nominee of scalars for event information transmittal. Extensive experiments have been accomplished to affirm the efficiency of our proffered method. We changed the number of cameras deployed (noc) and the number of scalars deployed (nos). The results attained from the experimental studies in terms of number of camera sensors activated (nca), coverage ratio (cr), redundancy ratio (rr), event loss ratio (elr) and energy expenditure for camera actuation (eeca) assert the superiority of our profferred approach over existing approaches.
\end{abstract}

Index Terms: Event Grade, Field of View, MCIM, MSIM, My Waiting List, Scalar Premier

\section{INTRODUCTION}

In this modern age of advanced technological procreation, the adoration of sensors is fundamentally owing to their dissonant perspective of relevance. However, such widespread applications give rise to diversified challenges. The most protrusive challenge in such networks is to attain an improved coverage of the area under consideration, while conjointly lowering the redundancy in data transmission which prevails owing to the superimposing of Field of Views (FoVs) of cameras. Consequently, the scalars residing at the overlapping zones convey the common information pertaining to the ongoing event to their concerned cameras as portrayed in Fig. 1.

The pink circle represents the event region. The tiny squares denoted by $\mathrm{C} 1, \mathrm{C} 2, \mathrm{C} 3$, etc. denote the camera sensors and the tiny filled circles denote the scalar sensors. Whenever, event takes place, it get ensnared through scalars and those scalars send the event information to the concerned camera sensors. Here, the triangular shapes represent the FoVs of the cameras. the scalars residing at superimposed areas of the FoVs report the same data

Revised Manuscript Received on September 14, 2019.

Sushree Bibhuprada B. Priyadarshini, Computer Science nd Information Technology, Siksha 'O' Anusandhan Deemed to be University, Bhubaneswar, Odisha, India number of times. As same data is reported several times, this results in redundant data transmittal, thereby leading to undesired energy and power expenses. Thus, the total repeated data transfer must be diminished so that only lowered number of camera sensors get turned on. The prime objective is that the activation of camera sensors has to be accomplished so that the amount of event region coverage get escalated conjointly with the depreciation of the repeated data transfer.

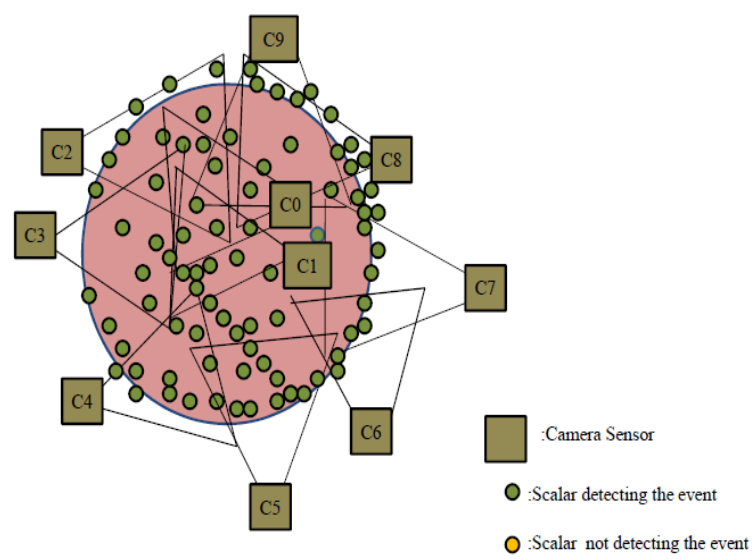

Fig. 1. A Scenario of Redundant Data Transmission

In this context, while thinking about the case of sensing environment efficient event tracking is both a popular and relevant aspect of investigation while talking about the case of a monitored area of examination as devised by Bhoi, S., etal.. in 2012 [1]. But, in case of failure of sensors it turns to be very hectic to track the prevailing event efficiently. Moreover, the major objective concerned in the method is to design a cluster through deploying density based clustering paradigm. Further, Bhoi, S., etal. (2012) [2] demonstrated another method while taking into account the case of wireless environment which employs a fault tolerance and conjointly achieves the required region coverage. Hence, for effective event tracking, sensors play a very significant role in ensuring reliability while monitoring the event zone under speculation.

In this paper, a new distributed scheme regarded as "Pentagonal Scalar Premier Neighborhood for Optimum Camera Actuation (PSPN-OA)" is demonstrated that elects scalar premiers among the scalars uniformly along all the directions of the virtual compartment. In this scheme, the

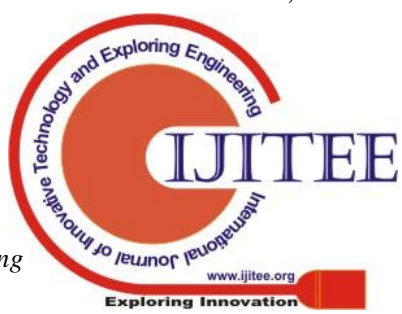


elected Scalar Premiers (SPS) act as the delineative of the scalar sensors that report about the prevailing event to the concerned camera sensors. The main aim of our proffered approach $P S P N-O A$ can be advocated as follows:

- The PSPN-OA approach divides the whole monitored area into number of smaller compartments known as Virtual Compartments (VCs). The proposed strategy engrosses the election of five $S P S$ in each of the VCs individually.

- $\quad$ The SP election is carried out such that the camera sensors which are actuated owing to the elected SPS can camouflage greater amount of different portions of the prevailing event region uniformly, while conjointly diminishing the camera activation in concerned monitored zone.

- The activation of reduced number of camera sensors in case of our proffered PSPN-OA method leads to reduced amount of redundant data transfer with lower amount of superimposing among the FOVs of actuated cameras..

- In addition, the amount of energy exhaustion for camera to be turned on is diminished in the proffered scheme owing to activation of minimal count of cameras.

The subsequent section elaborates the related work carried out in our field of study. Later on, we discuss the proposed scheme along with the whole methodology associated with the proposal. Subsequently, the results attained from the experimental investigation has been elaborately discussed. Finally, we conclude the paper with direction towards the future mission.

\section{RELATED WORK}

Various work have been carried out in past for lowering of the amount of repeated data communicated. In this connection, Distributed Collaborative Camera Actuation based on Scalar Count (DCA-SC) [3] represents one popular scheme for lowering the repeated data communicated. Here, the cameras collectively make decision regarding their activation. Likewise, the approach discussed in [4] devised a Non-Heuristic $(N-H)$ scheme aimed at lowering the Energy as well as Power expenditure prevailing in case of WMSNs. This strategy is focused on making the camera sensors activated unnecessarily in off condition and at the same time diminishing the amount of redundant data transmittal owing to such cameras.

Moreover, Distributed Collaborative Camera Actuation Scheme based on Sensing-Region Management (DCCA-SM) [5] represents a method which effectively segregates the whole region into a number of sensing zones. Afterwards, in every region, the cluster heads get determined those report the cameras about the happening of an event. Besides, the notion of directional coverage method [6] focuses on independent targets attached with differentiated importances. Moreover, the article addresses the "prioritybased target coverage" scheme. It attempts to select the minimal subset of directional sensors which ensnare all targets, thereby pacifying the expected priorities.

Similarly, S. Sundhar Ram (2007) [7] advocated a novel strategy of path coverage and the examination of the entire coverage phenomenon cajoled on 1-dimensional path through sensor network get modeled as a 2-dimensional Boolean model. Moreover, the strategy demonstrated in [8] that puts focus on directional cover sets issue of arranging the sensor directions into a gathering of various cover sets in the objective of prolonging the life-time. Paper [9] has given a strategy that advances in two passes for the goal of repeated data minimization. A local elimination has been accomplished which expels the repeated messages locally in every state of the automaton. Further, the approach proffered in [10] depicts a methodology where a method is proposed to estimate the similarity between the data gathered to the base station.

Apart from that, the approach [11] elaborates for sensor placement. Further, the proffered formulation assists in framing of larger Wireless Sensor Networks (WSN), which are established in effective way so that this preserves proper balance between lowering congestion while the data packets follow the shorter routes. Besides, a redundant positioning framework discussed in [12] depicts a new design for processing huge deal of data from various pervasive appliances. Moreover, a new fangled method has been given in [13] where provides desired $k$ coverage, where every point in a field get camouflaged through minimum $k$ number of sensors. The proffered scheme constructs maximal number of layers, in which every layer is 1 covered.

Besides, an indepth study has been carried out in [14] from perspective deformation domain. First of all, the deformation of the point is segregated into 3 parts. Again, noise immunity of every portion associated with several $F O V s$ is elaborated for justifying the prime reason behind the hecticness in the surveying of constricted cameras. Moreover, method given. in 2008, depicted a problem with the objective of prolonging the lifetime of network through scheduling [15] the sensors into various sets so that every sensor preserve target coverage as well as connectivity among all the active sensors and the destined sink. Further, the in [16] uses a cooperative multi-camera target ensnaring approach for those sensor networks, in which the target ensnaring [16] has been done through the sensors coordination based on the automatic node determination.

A view coverage method given in [17] estimates the coverage quality [17] along with a finer granularity for the objective of recognizing face. Similarly, the work carried out in [18] deploys a method which uses differential evolution optimizer as the search strategy. Likewise, the work in [19] used a dynamic itinerary for the certainty map. Furthermore, the approach in [20] has stated an analysis of various techniques concerned with coverage predicaments. Similarly, the method in [21] demonstrated the coverage goal as a measure of the quality of service that is afforded through a specific network construction. In like manner, a novel grid coverage methodology for efficient tracking and target position in distributed sensor networks is described in [22]. The sensor placement issue for a planar grid zone get formulated as the combinatorial optimization predicament as considered in [23] Further, the method is concerned with the

Blue Eyes Intelligence Engineering

\& Sciences Publication

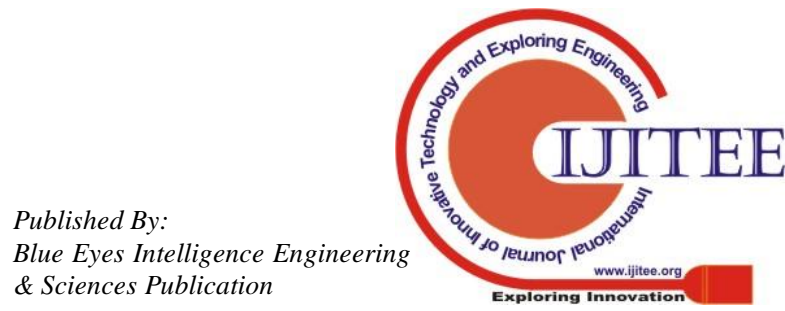


goal of prolonging the total detection probability.

A novel approach [24] that works efficiently for considering multiple events which occur concurrently in the geographic monitored zone, at the same time lowering the count of undesired activated cameras sensors thereby curtailing the amount of redundant data reporting. Similarly, a maximal far-flung methodology [25] uses a distributed method that involves maximum far-distant scalar premier election for camera to be turned on. This way selection reduces the possible superimposing among the FoVs, hence lowering the amount of repeated data transmittal prevailing due to it. Likewise, in another investigative work in [26] employs a distributed technique based on election of scalar leaders for the purpose of improved coverage of the occurring event zone. This manner of scalar leader determination is done such that the leaders get systematically arranged in a hierarchical manner where every child node is located minimum at a length of double of Depth of Fields (DoFs) chosen and the elected leaders act as the entity for event information transmittal to the sensors [26].

Despite all the aforesaid methods try to lower the amount of repeated data transfer, redundancy still persists in case of data transmittal. Hence, the goal is to activate merely the desired count of camera sensors so that the amount of repeated data transfer is reduced, while conjointly attaining enriched coverage of the concerned area.

\section{PROPOSED PSPN-OA METHOD}

This paper proffers a novel method known as "Pentagonal Scalar Premier Neighborhood for Optimum Camera Actuation (PSPN-OA)" which activates reduced number of cameras while conjointly affording enhanced event coverage while diminishing the redundant data transmittal.

Following are the steps encountered in our proffered $P S P N-O A$ method:

\section{Step 1. Random Placement of Sensors}

At the beginning all the scalars and cameras are arbitrarily deployed in the monitored zone. Initially, the ids of all the camera sensors get retained in a list namely, Current Waiting List $(C W L)$. A list namely Event Grade Id List $(E G I L)$ is preserved that maintains the ids of the camera sensors that finally undergo activation. Cameras and scalars broadcast My Camera Information Message (MCIM) and My Scalar Information Message (MSIM) successively that retain the id as well as position information of the respective sensors.

Just after the receipt of the two messages all the sensors become aware regarding the position and ids of all the sensors. Now each of the camera sensors finds its distance from scalar sensors individually. If the concerned Euclidian distance is lower than the DoFs of the corresponding camera; then it is considered that the particular scalar sensor comes under the purview of the concerned camera and hence the id of the particular scalar now get added to the id list of a table called Recent Field of View (RFOV) table.

\section{Step 2. Selection of Scalar Premiers (SPS)}

The monitored zone get divided into number of Virtual Compartments (VCs) for effective determination of Sps. The division is carried out following the same strategy as used in paper [25] such that the total monitored region of size $500 * 500$ get divided into $100 \mathrm{VCs}$. Further, within each of the virtual compartments (VCs), five SPs are determined among the scalars present within that particular $V C$ as portrayed in Fig. 2.

Such thing is accomplished so that the initial SP get selected towards the right side of the $X$-axis that passes through the centre of $V C$. Such premier is selected such that is the maximum distant scalar that resides within the particular virtual compartment at farthest distance from the centre of the particular $V C$ but within the concerned $V C$. If no such scalar sensor is available owing to random deployment of sensors; then the scalar sensor having sensing range spanning such virtual $X$-axis is determined as the initial premier. If no such scalar still covers the virtual axis, at that time the farthest scalar sensor that lies at maximal distance from the centre of $V C$ along the counter clockwise direction from concerned virtual axis get selected as the initial scalar premier.

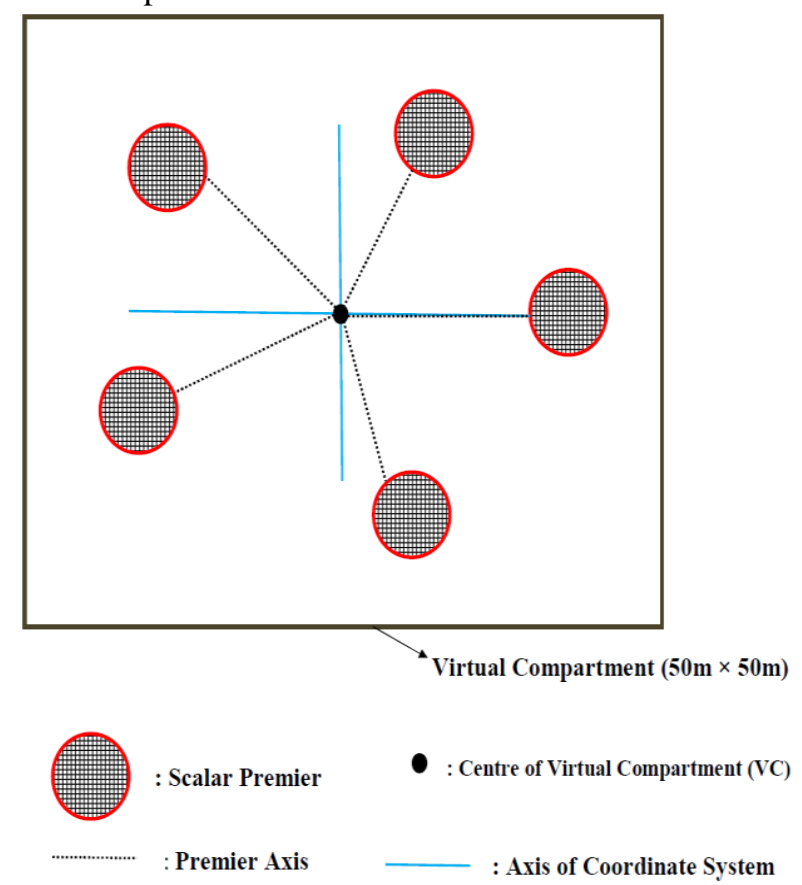

Fig. 2. A Scenario of Scalar Premier (SP) Determination

After the selection of the first SP gets over, the selection of the subsequent premiers commences. The subsequent premiers are selected such that they are elected at angles $72^{\circ}$ from the already selected scalar premiers such that every selected premier makes angle of approximately $72^{\circ}$ with its previously selected premier. However, as discussed earlier, there may not exist such scalar premiers exactly residing at the concerned angle. At such situation, the scalar whose sensing range spans the virtual axis along the concerned angle is determined as the $S P$ concerned to the desired axis. In this manner, all the premiers get selected along different directions. The angle $72^{\circ}$ is taken for uniform selection of scalar premiers along various directions such that the

Blue Eyes Intelligence Engineering

\& Sciences Publication 
cameras getting actuated owing them can camouflage the event region more effectively. In this way the SPS get selected in each of the $V C s$ of the region to be tracked.

\section{Step 3. Event Reporting and Camera Sensor Activation}

During the time of event occurrence, the elected SPS which come within the prevailing event area ensnare the event and inform about the ongoing event to the corresponding cameras within whose $R F O V$ table they lie by sending a DETECTION message that retains the concerned $S P$ 's $i d$ and the prevailing event information. Being reported from the $S P$; the camera sensor now updates its Event Reporting Scalar Premier List $(E R-S P L)$ that retains the ids of merely those SPS which ensnare the occurring event. In this context, the determination of event point and event radius by the scalar get accomplished by the following way:

(i) When any event occurs, the scalars that detect the event acquire signal having definite intensity. Again, the signal strength diminishes with rise in distance from the point of happening of the prevailing event. At the instant, a scalar attains a signal, it sends DETECTION message which retains the intensity of the signal which this got. Now, in like manner all scalar sensors can know the intensities of signals received by the other sensors. The scalar sensor can recognize the direction along which the signal intensity increases through exchange of information with its neighbors.

Following are the notations used:

\section{Notations Used:}

$I_{P O}$ : intensity of signal $(I O S)$ at the point of occurrence of event

$E R$ : radius of occurring event

$I_{O}: I O S$ at the point other than the occurring event point, at which any scalar $s$ is residing

$d_{I}$ : decelerating rate of the signal intensity

$p, q: p$ is the scalar sensor handy to the occurring event than scalar sensor $\mathrm{q}$

$U, \mathrm{~V}:$ IOSs acquired by $p$ and q respectively

$\Delta t$ : time appropriated by the signal to move from $p$ to $q$ which is indicated as follows:

$$
\text { Mathematically } \Delta t=t_{2}-t_{1} \text {, }
$$

$t_{1}$ and $t_{2}$ represent the time when the signals are acquired by $p$ and $q$ successively.

As $p$ is closer to the prevailing event than $q$, thus, the $I O S$ $(V)$ received by $q$ is lower than $I O S s(U)$ acknowledged by $p$ and can be interpreted as shown below:

$$
V=U-d_{I} \times \Delta t
$$

(since the decelerating rate of the signal intensity $d_{I}$ is negative)

Now, $d_{I}$ can be estimated by the following equation

$$
d_{I}=(U-V) / \Delta t
$$

Thereafter, the scalar computes the position of the other scalar (let it be point $N$ having coordinate $\left(X_{\mathrm{n}}, Y_{\mathrm{n}}\right)$ ), at which $I O S$ is the maximal from the received DETECTION message. This intensity is the value of $I_{P o}$.

Now, $I_{O}^{2}=I_{P O}:^{2}-2 d_{I} \times S_{1}$

where $I_{O} \leq I_{P O}$

$$
S_{1}=\left(I_{P O}:{ }^{2}-I_{O}{ }^{2}\right) / 2 d_{l} \text {, }
$$

(ii) Now the position of minimum signal strength accepting scalar get attained from by the DETECTION message attained through the various scalars. Let this point be $P$ having coordinate position $\left(X_{\mathrm{k}}, Y_{\mathrm{k}}\right)$. During the period of sprinkling of sensors, a threshold value for the IOS attained by the scalar sensor is set for determining whether the intensity of the signal received is significant for deciding that the event information to be tracked is needed or not.

(iii) Through DETECTION message the location of the scalar sensor which received the IOS value equal or just higher than the considered threshold value is selected. Let such point be $B$ having coordinate $\left(X_{\mathrm{B}}, Y_{\mathrm{B}}\right)$. The Euclidian distance from point $N$ to $B$ is the value of the event radius ER.

(iv) After determination of event point and event radius, all the camera sensors now calculate the sum of event informing SPs that lie within their DOFs known as Event Reporting Scalar Premier Estimate (ER-SPE). The estimation of $E R-S P E$ value is done as follows:

- Assign sum to zero (0)

- For each camera sensor a and any scalar premier $b$, estimate the Euclidian distance $\left(\right.$ Edist $\left._{a, b}\right)$ between them.

- If Edist $t_{a, b}<D O F$, then sum $=$ sum +1 ;

- For each camera sensor a, execute this calculation for every scalar premier $b$.

- Now update the sum value every time, if their corresponding Edist $t_{a, b}<D O F$ of corresponding camera sensor.

- $\quad$ Further, the updated sum value represents ER-SPE value of camera a.

* After the estimation of the ER-SPE value is over, the camera sensors append their ids to either Multi Premier Camera Ballot $(M P-C B)$ or Single Premier Camera Ballot $(S P-C B)$ based on the ER-SPE value. Further, $M P-C B$ and $S P-C B$ retain the ids of camera sensors in increasing sequence possessing $E R-S P E \geq 2$ and $E R-S P E=1$ successively. The ids for the cameras are kept in $M P-C B$ and $S P-C B$ in the same sequence as that of $C W L$.

* Now, the camera sensor whose id appears first in $M P-C B$ gets actuated first and its $i d$ is included in $A L$ and gets expunged from $C W L$.

* When a camera undergoes actuation, it transmits a Update Scalar Premier (USP) message to the remaining camera sensors. USP retains the ids of SPs residing within the DoFs of camera sensors, that are estimated based on the estimated Euclidian distance between the actuated camera sensor and the concerned $S P$.

* As soon as USP message is received, the Update Message id List (UM-IL) get updated which retains the ids of SPS contained in the corresponding USP message. Consequently, the subsequent camera coming immediately in the $M P-C B$ (say, $b$ ) matches the $i d s$ of SPs contained in its ERSPE with the ids of SPs maintained in USP message, transmitted by the actuated camera.

* Now the following scenario prevails:

Where, $S_{l}$ is the distance of event point from any sensor $s$. 
if (scalar premier ids retained by USP message and that maintained in ERSPL list match totally)

Then $\{$ No need to actuate the camera $b\}$

else

Then \{Camera $b$ has to be activated\}

* Likewise, the remaining cameras maintained successively in $M P-C B$ list decide to go to turned on condition based on comparing their corresponding $S P$ ids contained in their ERSPL list with the $S P$ ids contained in USP message based on the previous two situations.

* Whenever, a camera get actuated its id is included in $C A L$ and gets removed from the $C W L$. At the time when the corresponding cameras residing in $M P-C B$ list get actuated, then the ids of scalar sensors lying in USP messages of already actuated cameras, contained in $U M-I L$ for $M P-C B$ list are compared with the $S P$ ids contained in $E R S P L$ list of cameras lying in $S P-C B$ list. Further, in case the scalar $i d s$ contained in ERSPL of camera (s) lying in $S P$ $C B$ list completely matches with $S P$ ids retained in updated $U M-I L$, at that time no necessity to actuate the corresponding camera sensor.

* However, if a conflict is noticed, that camera pertaining to $S P-C B$ gets actuated. The actuated camera sensor of $S P-C B$ list broadcasts USP message.

* Such process persists till all the cameras decide either to be actuated or to be preserved in off condition. Thereafter, EGIL is updated. The total count of camera sensors lying in EGIL denotes the Event Grade.

\section{RESULTS AND DISCUSSION}

We have conducted the performance analysis of our proffered PSPN-OA method by taking results of three other approaches currently emerged recently [3, 4, 5]. They include: DCA-SC [3], N-H[4], DCCA-SM[5] methods and proffered PSPN-OA.

\section{Simulation Setting}

Our paper has been implemented using $C++$. During the experimentation, all the scalars sensors and camera sensors are considered to be arbitrarily sprinkled where as the camera sensors are considered to have predetermined fixed locations. Omni-directional camera sensors are used in case of our proffered strategy as they can trap panoramic image of objects uniformly. The number of cameras (noc) and number of scalars (nos) are varied independently and marked the impact on performance metrics as described below:

1. number of cameras actuated (nca)

2. eergy expenditure for camera actuation (eeca)

3. coverage ratio(cr): The cr is depicted as "the portion of the area of events that is covered by all the activated cameras with respect to the whole regions of the occurring events"[3].

4. event loss ratio (elr): The elr is depicted as "the portion of area of events that are not covered with respect to whole region of occurring events".

5. redundancy ratio( $\mathrm{rr}$ ): $\mathrm{rr}$ can be defined as "the ratio of total portion of superimposed portions of FOVs of actuated cameras to the total unique portions of event region that covered by the concerned cameras".

\section{Results Discussion}

\section{$>\quad$ Impact of variation noc}

* The noc was varied and its impact was marked on $n c a, r r, c r$, elr and eeca respectively in DCA-SC, N-H, DCCA-SM schemes as well as proffered PSPN-OA method as displayed in Table 1, Table 2, Table 3 and Table 4 successively. From all the tables it is crystal clear that with a rise in $n c d$, the $n c a$ gradually rises in case of all the approaches. Further, the value of $n c a$ is obtained to be the lowest in proffered PSPN-OA method.

* Likewise, with a hike in $n c d, c r$ hikes in the other schemes. It is owing to the cause that with rise in noc, greater count of camera sensors come under the ambit of prevailing event and hence greater number of cameras undergo actuation. Thus, greater portions of event area gets covered that gives rise to increasing value of coverage ratio.

* Moreover, the $\mathrm{cr}$ value is attained to be the maximum in proffered $P S P N-O A$ scheme and event area coverage is attained to be maximal of $86 \%$ in the proffered strategy.

* Similarly, with hike in noc, the $r r$ goes on rising in case of all the methods as with rise in noc, the amount of superimposing zone among the Fovs hikes in every scheme. Further, the redundancy in percentage obtained in the proffered method is the minimal at $39 \%$ while varying the $n c d$. Moreover, the eeca is found to be the minimal in proffered $P S P N-O A$ scheme than the other existing schemes as least count of camera sensors undergo actuation in case of the proffered scheme.

\section{Impact of variation of nos}

* The number of scalars has been varied and its effect has been marked on several performance metrics in case all the approaches as presented in Table 5, 6, 7 and 8 successively.

* The tables clearly depict that with a hike in $n s d$, the $n c a$ rises in all the approaches because with gradual rise in $n s d$, the count of event ensnaring scalars also goes on rising. Moreover, the nca is obtained to be the minimal in our proffered scheme.

* Similarly, with a hike in $n s d$, the value of $c r$ hikes in all the methodes and is attained to be the maximum in the proffered method. The initial rise in $c r$ is due to the fact that with rise in $n s d$ greater number of scalar premiers are going to ensnare the prevailing event. Further, maximal coverage of $92 \%$ and minimal event loss of $8 \%$ is attained in case of our PSPN-OA scheme.

* However, the $r r$ found in our proffered scheme is the minimal that ensures the effectiveness of our proffered $P S P N-O A$ scheme.

* With hike in number of scalar the eeca also increases. However, it is observed that eeca is obtained as the least in our proffered PSPN-OA method in comparison of others. 
Enhanced Event Coverage And Redundant Data Minimization Employing Pentagonal Scalar Premier Selection In Wireless Multimedia Sensor Networks (Wmsns)

Table 1. Impact of Varying noc in $D C A-S C$

\begin{tabular}{|l|l|l|l|l|l|}
\hline ncd & nca & cr & rr & elr & $\begin{array}{l}\text { eeca } \\
\text { (joule) }\end{array}$ \\
\hline $\mathbf{1 8 0}$ & 106 & 0.67 & 0.63 & 0.32 & 136.96 \\
\hline $\mathbf{2 1 0}$ & 111 & 0.69 & 0.66 & 0.31 & 142.08 \\
\hline $\mathbf{2 4 0}$ & 116 & 0.7 & 0.68 & 0.30 & 148.48 \\
\hline $\mathbf{2 7 0}$ & 118 & 0.71 & 0.71 & 0.29 & 151.04 \\
\hline $\mathbf{3 0 0}$ & 121 & 0.72 & 0.73 & 0.28 & 154.88 \\
\hline $\mathbf{3 3 0}$ & 123 & 0.73 & 0.74 & 0.27 & 157.44 \\
\hline
\end{tabular}

Table 2. Impact of Varying noc in $\mathrm{N}-\mathrm{H}$

\begin{tabular}{|l|l|l|l|l|l|}
\hline $\mathbf{n c d}$ & nca & cr & rr & elr & $\begin{array}{l}\text { eeca } \\
\text { (joule) }\end{array}$ \\
\hline $\mathbf{1 8 0}$ & 101 & 0.71 & 0.58 & 0.29 & 129.28 \\
\hline $\mathbf{2 1 0}$ & 104 & 0.73 & 0.62 & 0.27 & 133.12 \\
\hline $\mathbf{2 4 0}$ & 108 & 0.74 & 0.64 & 0.26 & 138.24 \\
\hline $\mathbf{2 7 0}$ & 109 & 0.76 & 0.65 & 0.24 & 139.52 \\
\hline $\mathbf{3 0 0}$ & 111 & 0.77 & 0.66 & 0.23 & 142.08 \\
\hline $\mathbf{3 3 0}$ & 113 & 0.78 & 0.67 & 0.22 & 144.64 \\
\hline
\end{tabular}

Table 3. Impact of Varying noc in DCCA-SM

\begin{tabular}{|l|l|l|l|l|l|}
\hline ncd & nca & cr & rr & elr & $\begin{array}{l}\text { eeca } \\
\text { (joule) }\end{array}$ \\
\hline $\mathbf{1 8 0}$ & 106 & 0.68 & 0.61 & 0.32 & 135.68 \\
\hline $\mathbf{2 1 0}$ & 108 & 0.70 & 0.64 & 0.30 & 138.24 \\
\hline $\mathbf{2 4 0}$ & 112 & 0.72 & 0.66 & 0.28 & 143.36 \\
\hline $\mathbf{2 7 0}$ & 115 & 0.73 & 0.69 & 0.27 & 147.20 \\
\hline $\mathbf{3 0 0}$ & 116 & 0.74 & 0.70 & 0.26 & 148.48 \\
\hline $\mathbf{3 3 0}$ & 118 & 0.75 & 0.72 & 0.25 & 151.04 \\
\hline
\end{tabular}

Table 4. Impact of Varying noc in proposed PSPNOA

\begin{tabular}{|l|l|l|l|l|l|}
\hline ncd & nca & cr & rr & elr & $\begin{array}{l}\text { eeca } \\
\text { (joule) }\end{array}$ \\
\hline $\mathbf{1 8 0}$ & 75 & 0.79 & 0.39 & 0.21 & 96.00 \\
\hline $\mathbf{2 1 0}$ & 78 & 0.81 & 0.41 & 0.19 & 99.84 \\
\hline $\mathbf{2 4 0}$ & 83 & 0.83 & 0.45 & 0.17 & 106.24 \\
\hline $\mathbf{2 7 0}$ & 88 & 0.84 & 0.47 & 0.16 & 112.64 \\
\hline $\mathbf{3 0 0}$ & 89 & 0.85 & 0.48 & 0.15 & 113.92 \\
\hline $\mathbf{3 3 0}$ & 92 & 0.86 & 0.50 & 0.14 & 117.76 \\
\hline
\end{tabular}

Table 6. Impact of Varying nos in N-H

\begin{tabular}{|l|l|l|l|l|l|}
\hline nsd & nca & cr & rr & elr & $\begin{array}{l}\text { eeca } \\
\text { (joule) }\end{array}$ \\
\hline $\mathbf{1 6 0}$ & 95 & 0.69 & 0.60 & 0.31 & 121.6 \\
\hline $\mathbf{2 0 0}$ & 99 & 0.71 & 0.63 & 0.29 & 126.72 \\
\hline $\mathbf{2 4 0}$ & 102 & 0.72 & 0.65 & 0.28 & 130.56 \\
\hline $\mathbf{2 8 0}$ & 104 & 0.74 & 0.67 & 0.26 & 133.12 \\
\hline $\mathbf{3 2 0}$ & 105 & 0.75 & 0.68 & 0.25 & 134.40 \\
\hline $\mathbf{3 6 0}$ & 108 & 0.76 & 0.69 & 0.24 & 138.24 \\
\hline
\end{tabular}

Table 7. Impact of Varying nos in DCCA-SM

\begin{tabular}{|l|l|l|l|l|l|}
\hline nsd & nca & cr & rr & elr & $\begin{array}{l}\text { eeca } \\
\text { (joule) }\end{array}$ \\
\hline $\mathbf{1 6 0}$ & 100 & 0.66 & 0.42 & 0.34 & 128 \\
\hline $\mathbf{2 0 0}$ & 103 & 0.68 & 0.45 & 0.32 & 131.84 \\
\hline $\mathbf{2 4 0}$ & 106 & 0.70 & 0.47 & 0.30 & 135.68 \\
\hline $\mathbf{2 8 0}$ & 110 & 0.71 & 0.50 & 0.29 & 140.80 \\
\hline $\mathbf{3 2 0}$ & 112 & 0.72 & 0.52 & 0.28 & 143.36 \\
\hline $\mathbf{3 6 0}$ & 113 & 0.73 & 0.53 & 0.27 & 144.64 \\
\hline
\end{tabular}


Table 8. Impact of Varying nos in proposed PSPN-

\begin{tabular}{|l|l|l|l|l|l|}
\hline nsd & nca & cr & rr & elr & $\begin{array}{l}\text { eeca } \\
\text { (joule) }\end{array}$ \\
\hline $\mathbf{1 6 0}$ & 85 & 0.85 & 0.43 & 0.15 & 108.80 \\
\hline $\mathbf{2 0 0}$ & 88 & 0.86 & 0.44 & 0.14 & 112.64 \\
\hline $\mathbf{2 4 0}$ & 92 & 0.88 & 0.45 & 0.12 & 117.76 \\
\hline $\mathbf{2 8 0}$ & 97 & 0.90 & 0.47 & 0.10 & 124.16 \\
\hline $\mathbf{3 2 0}$ & 98 & 0.91 & 0.48 & 0.09 & 125.44 \\
\hline $\mathbf{3 6 0}$ & 99 & 0.92 & 0.50 & 0.08 & 126.72 \\
& & & & & \\
\hline
\end{tabular}

\section{CONCLUSIONS AND FUTURE SCOPE}

This article depicts a newfangled method known as $P S P N-O A$ that actuates minimal count of cameras at the same time conjointly diminishing the amount of repeated data reporting. Such selection of scalar premiers avoids multi-event informing by all the scalar sensors. Moreover, the uniform selection of scalar premier give rise to more even coverage of region tracked. Further, extensive experiments have been conducted to assess the effectiveness of our proffered scheme while assessing it with other existing approaches in the literature. The noc and nos were varied independently and the aftereffect were marked successively in case of all the methods. The numerical results make it obvious that the nca and eeca are attained as the minimal in our proffered PSPN-OA method than the other methods. Likewise, the $c r$ is found to be the maximal while the $r r$ is the minimal in case of our proffered PSPN$O A$ method. While varying the noc, it is clear that the event coverage is attained to be the maximum at $86 \%$ in proffered method. Likewise, while varying the noc the minimized value of $r r$ and $e l r$ are obtained as $39 \%$ and $14 \%$ respectively in our proffered method. Similarly,during the variation of nos, the event coverage is found to be the maximal in case of our approach at $73 \%$ where as the redundancy and event-loss are marked to be minimal at $42 \%$ and $34 \%$ respectively.

Although our proposed system reduces the amount of redundant data transmission, still it has some limitations. We have used camera sensors those are having fixed positions by keeping the real life random deployment scenario of sensors in mind. However, as a direction towards our future work, mobility can be added to the cameras for increasing the adaptability of entire system. Further, we are planning to map our proposed system to three dimensional scenarios as an insight towards the future investigation.

\section{ACKNOWLEDGEMENT}

The author is highly grateful to the Institute of Technical Education and Research, Siksha 'O' Anusandhan Deemed to be University for affording the necessary amenities and desired assistance for the research.

\section{REFERENCES}

1. S., Bhoi, S. Panda, P. Khilar, "A density-based clustering paradigm to detect faults in wireless sensor network". IInternational Conference on Advances in Computing, Springer, 2012, pp. 865-871.

2. S. Bhoi, S. Panda, P. Khilar, "A network survivability approach to resist access point failure", Second International Conference on Internet Computing and Information Communications, Springer,2012, pp. 293-30.

3. A. Newell, K. Akkaya, "Distributed Collaborative Camera Actuation for Redundant Data Elimination in Wireless Multimedia Sensor Networks", Ad Hoc Networks, Elsevier, 2011, 9 (4), pp. 514-527.

4. S. B. B. Priyadarshini, S. Panigrahi,"A non-heuristic approach for minimizing the energy and power consumption in wireless multimedia sensor networks", International Conference on Computational Intelligence and Networks (CINE),.IEEE, 2015, pp. 104-109.

5. W. Luo, Q. Lu, J. Xiao,'Distributed collaborative camera actuation scheme based on sensing-region management for wireless multimedia sensor networks" Int. J. Distrib. Sensor Netw. 2012, pp.1-14 ,ArticleID:486163,doi:10.1155/2012/486163 http://www.hindawi.com/journals/ijdsn/2012/486163/ref/

6. 6. J. Wang, C. Niu, R. Shen, "Priority-based target coverage in directional sensor networks using a genetic algorithm", Computers and Mathematics with Applications ,Elsevier, 2009, 57(11-12), pp. 1915-1922).

7. S. Sundhar Ram, D. K. Manjunath, S. Iyer, S., Y. Yogeshwaran, "On the Path Coverage Properties of Random Sensor Networks", IEEE TRANSACTIONS ON MOBILE COMPUTING, 2007, 6(5), pp. 494-506.

8. Yanli Cai, Wei Lou, Minglu Li and Xiang-Yang Li , “ Target-Oriented Scheduling in Directional Sensor Networks", IEEE INFOCOM 2007 proceedings, 2007, pp. $1550-1558$

9. A. Girault, A., "Elimination of redundant messages with a two-pass static analysis algorithm", Parallel Computing, Elsevier, 2002, 28(3), pp. 433-453.

10. A. Ghaddar, T. Razafindralambo,T., S. Tawbi, A Hijazi, "Algorithm for data similarity measurements to reduce data redundancy in wireless sensor networks", IEEE International Symposium on A World of Wireless, Mobile and Multimedia Networks (Wow Mom), ISBN: 978-1-4244-7264-2, June 14-june 17 2010, pp. 1-6, Montreal, QC, Canada.

11. S. Toumpis, S., T. Tassiulas, "Optimal deployment of large wireless sensor networks", IEEE Transactions on Information Theory, 2006, 52(7), pp. 2935-2953.

12. X. Han, X. Cao, E. Lloyd, C. Shen ,'Deploying directional sensor networks with guaranteed connectivity and coverage", Paper presented at $5^{\text {th }}$ Annual IEEE Communications Society Conference on Sensor, Mesh and Adhoc Communications and Networks, June 16 June 20 2008, San Francisco, CA, pp. 153-160

13. A. Abdullah, Al-Shalabi, M. Manaf, "DKCS-An efficient dynamic K- coverage scheduling Algorithm for Wireless Sensor Networks", International Symposium on Telecommunication Technologies (ISST), IEEE , 26-28 November, 2012, Kuala Lumpur, , pp. 94-99.

14. X. Yang, S.F. , "Effect of Field of view on the accuracy of camera calibration", Optik - International Journal for Light and Electron Optics, Elsevier, 2014 125(2), pp. 844-849.

15. Q. Zhao, M.G., "Lifetime maximization for connected target coverage in wireless sensor networks", IEEE/ACM 

Wireless Multimedia Sensor Networks (Wmsns)

Transactions on Networking (TON), 2008, 16(6), pp. 1378-1391.

16. Y. Wang, D. Wang, Wu Fang, "Automatic Node Selection and Target Tracking in Wireless Camera Sensor Networks", Computers and Electrical Engineering, 2014, 40(2), pp. 484-493.

17. C. Yang, W. Zhu, J. Liu, L. etal. "Self-orienting the Cameras for Maximizing the View Coverage Ratio in Camera Sensor Networks", Pevasive and Mobile Computing, Elsevier, 2015 17, pp. 102-121.

18. S. Sengupta, S. Das, M. D. Nasir, B. K. Panigrahi, "Multi-objective Node Deployment in WSNs: in Search of an Optimal Trade off among Coverage, Lifetime, Energy Consumption and Connectivity", Engineering Applications of Artificial Intelligence, Elsevier, 2013, 26(1), pp.405-416.

19. M. Karakaya, H. Qi., "Target Detection and Counting using a Progressive Certainty Map in Distributed Visual Sensor Networks", Distributed Smart Cameras, ICDSC 2009, Third ACM/ IEEE International Conference, pp. 18.

20. Younis and Akkaya, "Strategies and Techniques for Node Placement in Wireless Sensor Networks: a Surve". Ad hoc Networks, Elsevier, 2008, 6(4), pp. 621-655.

21. S. Meguerdichian, F. Koushanfar, M. Potkonjak,,M. Srivastava, "Coverage Problems in Wireless Ad-hoc Sensor Network", IEEE Infocom 3,2001, pp. 1380-1387.

22. K. Chakrabarty, S. ,Iyengar, etal. , "Grid Coverage for Surveillance and Target Location in Distributed Sensor Networks", IEEE Transactions on Computers, 2002, 51(12), pp.1448-1453.

23. Q. Wu, S. V., Nageswara, etal., "On Efficient Deployment of Sensors on Planar Grid", Elsevier, 200730 (14-15), pp.2721-2734.

24. S. B. B. Priyadarshini, S. Panigrahi, "Centralised cum sub-centralised scheme for multi-event coverage and optimum camera activation in wireless multimedia sensor networks", IET Networks, Available in IEEE Xplore Digital Library, 2015, 4(6), pp. 314-328.

25. S. B. B. Priyadarshini, S. Panigrahi, "A Distributed Approach based on Maximal Far-flung Scalar Premier Selection for Camera Actuation" ICDCIT, Lecture Notes in Computer Science, Springer, 2016, pp. 87-91.

26. S. B. B. Priyadarshini, S. Panigrahi, "A Distributed Approach based on Hierarchical Scalar Leader Selection for Enhanced Event Coverage in Wireless Multimedia Sensor Networks", 13th International Conference, ICDCIT 2017, Lecture Notes in Computer Science, Springer, Bhubaneswar, India, January 13-16, 2017, pp. 3-14.

\section{AUTHOR'S PROFILE}

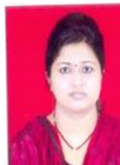

Dr. Sushree Bibhuprada B. Priyadarshini (Ph . D in Computer Science and Engineering) is currently working as an Asst. Prof. in Dept. of Computer Science and Information Technology at

Institute of Technical Education and Research (ITER), Siksha "O" Anusandhan, Deemed to be

University. She is the recipient of Orissa State Talent Scholarship and NRTS scholarship. She has got the certificate of Merit in Brigade Talent Search Exam. She has received the "All Round the best Student of the year" in 2005 from N. S. Police High School. She has secured the highest marks in Computer Science and Data Processing (CSDP) from ITER in 2011-13. She has been awarded for "Best Poster Presentation" in the Workshop on "Monitoring of Research of Ph. D. Scholars (MRS-2017)" during March 17-18 at VSSUT, Burla (An Event of Diamond Jubilee Celebration 1956-2016). She has received the "Best Student Paper Award" in 15th International Conference on Information Technology, IEEE organized by IIIT, Bhubaneswar, December 22-24, 2016. She has been awarded the "Best Poster Presentation Award" in Workshop on: "Women in Smart Computing", organized at KIIT, Bhubaneswar, December 2017.
She has been invited and selected as the Keynote Speaker of International Conference of Innovative Applied Energy, St. Cross College, Oxford University. Besides, She is the Member of IAENG, SDIWC, CSTA. She is also working as reviewer in International Journal of Engineering Research \&amp; Technology and IEEE Consumer Electronics journal. She has around 26 publications in various international journals and Conferences including IET, IEEE, Elsevier, Springer, IGI Global etc. 